\title{
I-band Cepheid Distance to WLM
}

Myung Gyoon Lee ${ }^{1}$, Wendy L. Freedman ${ }^{1}$, Barry F. Madore ${ }^{2}$

${ }^{1}$ Carnegie Observatories, Pasadena, USA, ${ }^{2} I P A C / J P L / C a l t e c h$, Pasadena, USA

WLM (DDO221) is a highly resolved dwarf irregular galaxy in the Local Group. Fifteen Cepheids in this galaxy have been studied by Sandage \& Carlson (1985, AJ, $90,1464)$ using photographic photometry. We have obtained $B V R I C C D$ photometry of stars in the central area $\left(2.2^{\prime} \times 3.5^{\prime}\right)$ of WLM. Figure 1 shows an $I-(V-I)$ diagram of $\sim 2,600$ measured stars including five known Cepheids. The distance to WLM has been estimated using the random-phase $I$ band photometr $y$ of five Cepheids ( $\mathrm{V} 7(\mathrm{I}=20.52), \mathrm{V} 29(\mathrm{I}=20.77), \mathrm{V} 48(\mathrm{I}=20.43), \mathrm{V} 66(\mathrm{I}=21.19), \mathrm{V} 67(\mathrm{I}=21.14))$ combined with their periods given by Sandage \& Carlson. We have obtained

$\Delta(m-M)_{I}(\mathrm{WLM}-\mathrm{LMC})=6.25 \pm 0.11$ and $(m-M)_{0}=24.92 \pm 0.21$, adopting $(m-M)_{0}(\mathrm{LMC})=18.5$ and $A_{I}(\mathrm{WLM})=0.04$. The distance to WLM has also been estimated independently using the brightness of the tip of the red giant branch (RGB) of low mass stars, following the method described in Da Costa \& Armandroff (1990, AJ, 100, 162). The tip of the RGB is detected at $I=20.85 \pm 0.1 \mathrm{mag}$. This estimate yields a distance modulus of $(m-M)_{0}=24.81 \pm 0.15$, which is in excellent agreement with the Cepheid distance modulus. Taking the average of Cepheid and TRGB distances, we obtain $\mathrm{M},(m-M)_{0}=24.87 \pm 0.08(940 \pm 30 \mathrm{kpc})$.

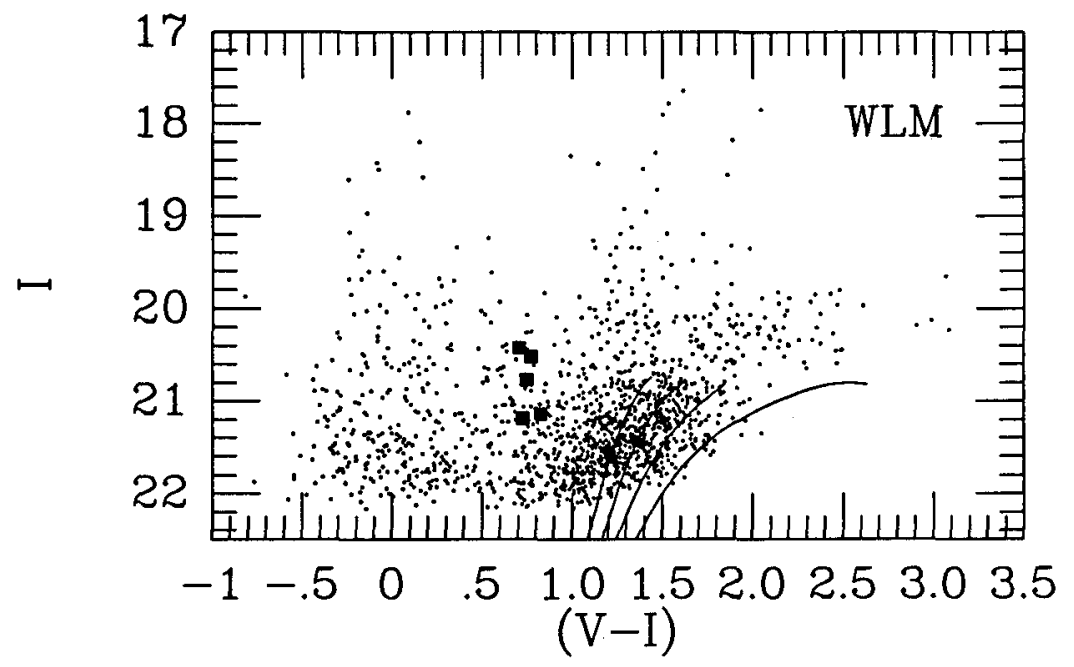

Fig. 1: $I-(V-I)$ diagram of $\sim 2,600$ measured stars in WLM. The filled squares represent the Cepheids. Note that there is a clearly distinguishable concentration of faint red stars with $I<20.85 \mathrm{mag}$, which represents the red giant branch (RGB) of low mass stars. The solid lines show the loci for the RGB of Galactic globular clusters, M15, M2, NGC 1851, and 47 Tuc (from Da Costa \& Armandroff 1990). 